\title{
A system for gene cloning and manipulation in the yeast Candida glabrata
}

(AIDS; Immunocompromised; mutant; pathogenic fungi; plasmid; Torulopsis; transformation)

\author{
Pengbo Zhou, Mark S. Szczypka, Rachel Young and Dennis J. Thiele \\ Department of Biological Chemistry, University of Michigan Medical School, Ann Arbor, MI 48109-0606. USA
}

Received by J.A. Gorman: 4 April 1993; Revised/Accepted: 22 September/5 October 1993; Received at publishers: 10 January 1994

\section{SUMMARY}

The opportunistic pathogenic yeast, Candida (Torulopsis) glabrata, is an asexual imperfect fungus that exists largely as a haploid. Besides being a clinically important pathogen, this yeast also provides a model system for understanding basic biological mechanisms such as metal-activated metallothioncin-encoding gene transcription. To facilitate molccular genetic studies in $C$. glabrata, we isolated a strain auxotrophic for uracil biosynthesis. The ura mutation could be functionally complemented by the URA3 gene of Saccharomyces cerevisiae, consistent with a defect in the $C$. glabrata $U R A 3$ gene in this strain. We also found that the centromere-based $S$. cerevisiae plasmid pRS316 could stably transform and replicate in multiple copies in $C$. glabrata. In contrast, high-copy-number $S$. cerevisiae plasmids containing the $2 \mu$ circle autonomous replication sequence were not able to replicate productively in C. glabrata. We cloned the C. glabrata $U R A 3$ gene, encoding orotidine-5' -phosphate decarboxylase, by complementation of a $u r a 3^{-}$strain of $S$. cerevisiae. The deduced amino-acid sequence is highly similar to that of the URA3 protein from S. cerevisiae. C. glabrata URA3 provides a genetic locus for targeted gene integration in $C$. glabrata. Integrative plasmids were constructed based on the cloned $C$. glabrata URA3 and are applicable for directed insertions of genes of interest at the ura 3 locus through homologous recombination.

\section{INTRODUCTION}

Candida (Torulopsis) glabrata is among several clinically isolated yeast species of the Candida genus.

Correspondence to: Dr. D.J. Thiele, Department of Biological Chemistry, University of Michigan Medical School, Ann Arbor, MI 48109-0606, USA. Tel. (1-313) 763-5717; Fax: (1-313) 763-4581; e-mail: Dennis_Thiele@UM.CC.UMICH.EDU

\footnotetext{
Abbreviations: $A$, absorbance ( $1 \mathrm{~cm}$ ); aa, amino acid(s); Ap, ampicillin; $A R S$, autonomously replicating sequence(s); bp, base pair(s); $C$., Candida; E., Escherichia; EMS, ethyl methanesulfonate; 5-FOA, 5-fluoroorotic acid; GCG, Genetics Computer Group (Madison, WI, USA); K., Kluyveromyces; kb, kilobase(s) or 1000 bp; MCS, multiple cloning site(s); $M T-H a$, tandemly amplified genomic locus encoding one of the MT-II isoforms of the C. glabrata metallothionein gene family; nt, nucleotide(s); ORF, open reading frame; $S$., Saccharomyces; $\mathrm{SC}$, synthetic complete media; SC-ura, SC lacking uracil; $U R A 3$, gene encoding orotidine- 5 -phosphate decarboxylase; wt, wild type; XGal, 5-bromo-4-chloro-3-indolyl- $\beta$-D-galactoside; YPD, rich media containing $1 \%$ yeast extract $/ 2 \%$ peptone/ $2 \%$ dextrose.
}

Although less virulent than Candida albicans, C. glabrata has been demonstrated to be the pathogen of opportunistic infections such as vaginitis, endophthalmitis and persistent Torulopsis glabrata fungaemia (Redondo-Lopez et al., 1990; Odds, 1987; Damani and Webb, 1988). Clinically, this species has been found in many human or animal organs including the gastrointestinal and genitourinary tracts, stomach, skin and urinary tract (White, 1976; Frye et al., 1988; Sinnott et al., 1987). C. glabrata is the second most frequently isolated species from vagina in both asymptomatic females and patients suffering from yeast vaginitis (Redondo-Lopez et al., 1990). Recently there have been increasing concerns over $C$. glabrata because it can cause systemic infections in immunosuppressed patients, where it may be life-threatening. $C$. glabrata infections were reported to be a factor in the death of diabetes (Hickey et al., 1983) and cancer patients (Aisner et al., 1976), and Moniaci et al. (1988) reported 
a case of $C$. glabrata infection in a patient with AIDS. Studies of the body's defense against Candida infection reveal that the human tumor neurosis factor $\alpha$ (TNF- $\alpha$ ) can enhance the fungicidal activity against $C$. glabrata, a process mediated by human neutrophils (Ferrante, 1989). However, the molecular mechanism of TNF- $\alpha$ in stimulating this potential antifungal activity of neutrophils remains to be determined. Because of the metabolic similarities between fungi and their eukaryotic hosts, efforts to develop efficient antifungal agents are largely hampered.

In order to study the molecular mechanisms underlying pathogenic as well as other biological processes of C. glabrata, the development of genetic tools and molecular biology methods is essential. The advantage of using C. glabrata to study the pathogenicity of the Candida species is that this organism is unicellular and exists as a haploid (Whelan, 1987), rendering it more tractable for the isolation of mutants and for manipulation of the $C$. glabrata genome. Recently, a gene cloning system using a DNA fragment carrying a C. glabrata autonomous replication function has been described (Mehra et al., 1992).

The aim of the present study was to develop genetic tools that can be used for gene manipulation in C. glabrata, and these include ( 1 ) the isolation of a $C$. glabrata mutant strain harboring a uracil biosynthetic mutation in the ura 3 locus, (2) the isolation and sequencing of the C. glabrata URA3 gene, which provides a genetic locus for targeted gene integration as demonstrated with the C. glabrata AMT1 gene and (3) the use of episomal plasmids that can conveniently shuttle between $C$. glabrata, S. cerevisiae and Escherichia coli.

\section{EXPERIMENTAL AND DISCUSSION}

\section{(a) Isolation of a ura3- auxotrophic strain and cloning of the C. glabrata URA3 gene}

To generate a $C$. glabrata strain suitable for genetic transformation, a strain bearing a mutation in the gene encoding orotidine 5 - phosphate decarboxylase was isolated. The C. glabrata strain $(85 / 038)$ was described previously (Zhou and Thiele, 1991) and used as the parental wt strain (a gift from Dr. P. Magee). One stable $u \mathrm{ra}^{-}$ mutant was identified after EMS mutagenesis, purified for single colonies and designated 'Q' (Fig. 1). The ura' phenotype of the $\mathrm{Q}$ mutant strain can be complemented by the $S$. cerevisiae URA3 gene on an episomal plasmid pRS316 as described in section $\mathbf{b}$, or in a single integrated copy (Zhou et al., 1992), consistent with a mutation in the $C$. glabrata orotidine-5'-phosphate decarboxylaseencoding gene, which is functionally equivalent to $U R A 3$ from S. cerevisiae (Rose et al., 1984).
To obtain a genetically defined locus for gene targeting by homologous recombination, a genetic selection approach was implemented to isolate the $C$. glabrata $U R A 3$ gene by complementation of a $S$. cerevisiae ura $3^{-}$ strain. This selection is based on the suggestion that $C$. glabrata and $S$. cerevisiae are very close evolutionarily (Barns et al., 1991) and that C. glabrata genes can function properly in S. cerevisiae (Zhou and Thiele, 1991; Zhou et al., 1992). A C. glabrata genomic DNA library was constructed and was transformed into the recipient S. cerevisiae strain DTY7 (leu2-3,-112 and ura3-52). A plasmid, designated U1(b), was recovered based on its ability to complement the ura $3^{-}$phenotype of DTY7. Restriction analysis indicated that U1(b) contains a $3.7-\mathrm{kb}$ insert. Furthermore, subcloning and complementation studies identified a $1.0-\mathrm{kb}$ XhoI-SspI fragment within the $3.7-\mathrm{kb}$ insert that enables DTY7 cells to grow on SC-ura media, suggesting that the functional C. glabrata URA3 gene is located within this fragment (Fig. la). The nt sequence of the $C$. glabrata URA3 gene was subsequently determined and a continuous ORF was identified using the GCG computer program (Fig. 1b). The $C$, glabrata URA3 gene encodes a polypeptide of 256 amino acids that shares extensive homology with the $U R A 3$ gene product from $S$. cerevisiae, demonstrating $82 \%$ protein sequence identity (Rose et al., 1984). Fig. 2 shows the aa sequence comparison between $C$. glabrata and $S$. cerenisiae URA3 proteins. Furthermore, the $C$. glabrata URA 3 protein is $85 \%$ and $74 \%$ identical to the functionally analogous proteins from the yeasts Kluyveromyces lactis and C. albicans, respectively (Shuster et al., 1987; Ernst and Losberger, 1989). This protein sequence similarity provides a basis for effective complementation of the $S$. cerevisiae ura3- auxotroph by $C$. glabrata URA3 gene product and vice versa (Zhou et al., 1992).

\section{(b) Episomal plasmids for gene transfer in $C$. glabrata}

The ability to stably introduce genetic information into C. glabrata by plasmid-mediated transformation is critical to the development of this organism as a facile experimental system. We observed that the low-copy centromere-based S, cerevisiae plasmid pRS316 (Sikorski and Hieter, 1989) can efficiently transform C. glabrata $Q$ $\left(\right.$ ura $^{-}$) strain, forming large colonies on SC-ura media after one to two days of incubation. This plasmid contains the ARS4, URA3 and CEN6 sequences of S. cerevisiae and exists as a single or low-copy episomal plastnid in baker's yeast (Sikorski and Hieter, 1989). Plasmid pRS316 can be readily recovered from $C$. glabrata by transforming total genomic DNA of the $C$. glabrata recipient cells into $E$. coli and isolating plasmid DNA from individual Ap-resistant E. coli transformants. This suggests that pRS316 exists in C. glabrata Q cells as an extra- 
a

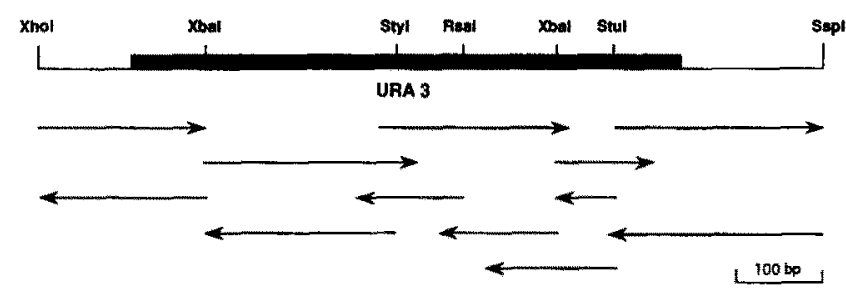

b

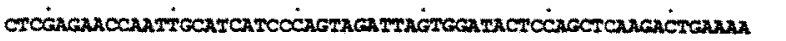

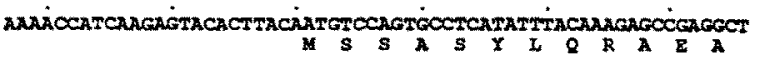

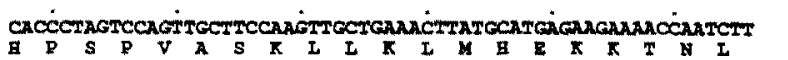

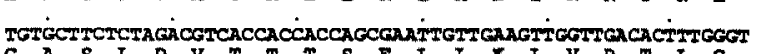

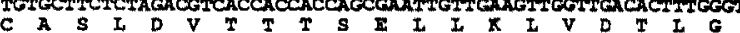
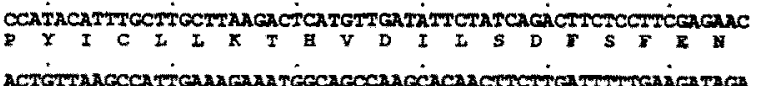

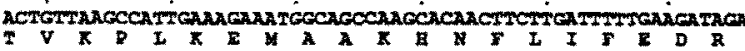

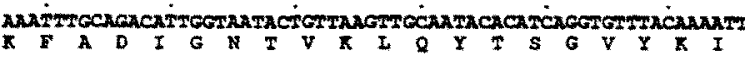

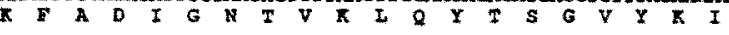

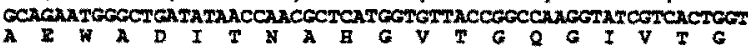

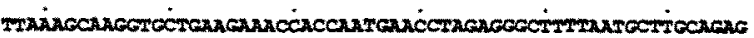

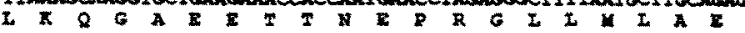

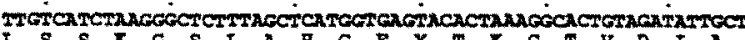

For
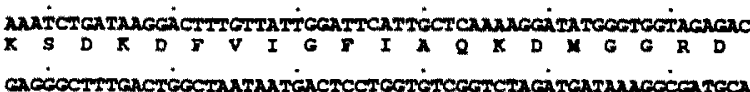

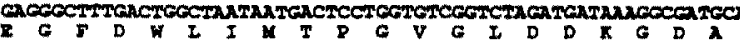

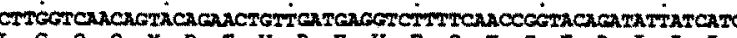

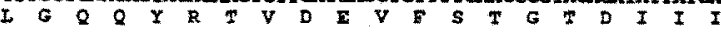

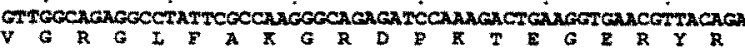

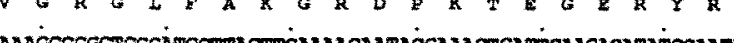

$X$ A $G$ W D A Y I X R I G W

COAACCTACA TCATACCTACCAACATCATCTTACAN CCCAATTGTACATTETCACUAAT

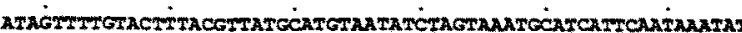

r

Fig. 1. C. glabrata ura $3^{-}$mutant strain and the URA3 gene. C. glabrata $85 / 038$ was treated with EMS to $60 \%$ survival. The auxotrophic mutant cells were enriched by Nystatin treatment as described (Haas et al., 1990) and were selected on 5-FOA plates for uracil auxotrophy (Ausubel et al., 1987). The ura $3^{-}$cells were purified to single colonies. Their ura $3^{-}$phenotype was complemented by introducing a $C$.glabrata episomal plasmid pRS316, which carries the $S$. cerevisiae URA3 gene functional in $C$. glabrata (Zhou et al., 1992). Transformation of $C$. glabrata was carried out using the lithium acetate transformation procedure, as described (Sherman et al, 1983). Competent cells for transformation were freshly prepared logarithmic phase cells grown in YPD medium (Sherman et al, 1983) with an $A_{600}$ between 2.0 and 3.0 . Routinely, we obtained approx 1000 transformants using $1.0 \mu \mathrm{g}$ of pRS316 plasmid DNA. To clone the C. glabrata URA3 gene, $C$. glabrata genomic DNA was partially digested with Sat $3 A$ and $20.0 \mu$ gere used for ligation into the BamHI site of the $S$. cerevisiae high-copy plasmid pRS425 carrying the $L E U 2$ gene as a selection marker (Sikorski and Hieter, 1989). $1 \%$ of the ligation mix was used to transform competent E. coli strain XL-1 blue (Stratagene, La Jolla, CA, USA), and the percentage of recombinant plasmids was determined by comparing the number of white colonies with the total number of transformants on LB plates containing XGal and $50 \mu \mathrm{g} \mathrm{Ap} / \mathrm{ml}$. We routinely obtained from $30 \%$ to $50 \%$ insertion frequency. A total of 30 individual colonies containing the plasmid-borne Sau3A DNA resiriction fragments of $C$. glabrata
MSSASYLQRAEAHP SPVASKLLKLMHEKXTNLCASLDVTTTSELLKLVDT 50 $11.1 .1: 11 \ldots 111111.11:-11111.11111111111 .111 .11:$ MSKATYKERAATHP SPVAAKLFN IMHEKOTNLCA SLDVRTTKELLELVEA 50

LGPY ICLLKTHVD I LSDF SËENTVKP LKEMAAKHNFL IFEDRKEAD IGNT 100

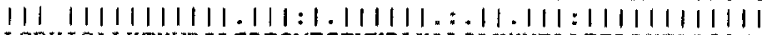
LGPKICLLKTHVDILTDF SMEGTVKP LKALSARYNELLFEDRKFAD IGNT 100

VKLQYTSGVYKIAEWAD I TNAHGVTGQG IVTGLKQGAEE TTNEPRGLLML 150 \|\|$\|\ldots\|\|:\|\|\|\|\|\|\|\|\|-1 .\|\| \cdot\|\|\|=11.1 .1\|\|\|\|\|$ VKLQYSAGVYRIAEWAD I TNAHGVVGPGIVSGLKOAAEEVTKEERGLLML 150

AELS SKGSLAHGEYTKGTVDIAKSDKDFVIGE IAOKDMGGRDEGFDWLIM 200

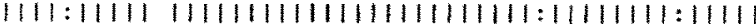
AELSCKGSLATGEYTKGTVDIAKSOKDFVIGEIAORDMGGRDEGYDWLIM 200

TPGVGLDDKGDALGQOYRTVDEVESTGTD IIIVGRGLFAKGRDRKTEGER 250

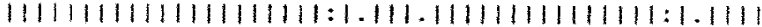
TEGVGLDDKGDALGQOYRTVDDVVSTGSOI I TVGRGLEAKGRDAKVEGER 250

YRKAGWDAYLKRIGN

$11111: 111: 1.1$

c. glabrata URA3

265

YRKAGWEA YLRRCGQQN

s. cerevisiae URA3

267

Fig. 2. Amino-acid sequence comparison between the URA3 proteins from C. glabrata (265 aa) and S. cerevisiae (267 aa) (Rose et al., 1984) using the GAP comparison of the GCG program. A vertical line indicates identical aa a colon (:) or a period () indicate aa with an evolutionary comparison values greater than 0.5 or $0.1-0.5$, respectively.

genomic DNA were grown to prepare plasmid DNA (Ausubel et al., 1987), and the size of the inserts was determined by digestion with $E c o R I+X b a I$ to release the cloned fragments, and electrophoretically fractionated on $1 \%$ agarose gels. The ligation mix used for yeast transformation had a $40 \%$ frequency of DNA fragment inscrtion and the average size of the inserts was approx. $3 \mathrm{~kb}$. Transformation of the ligation mixture into the recipient $S$. cerevisiae DTY7 strain (leu2-3,112 and ara3-52) yielded a total of 40000 colonies which grew on synthetic complete (SC) agar lacking leucine. This represents approx. 3 C. glabrata genome equivalents, based upon the average insert size, the percentage of recombinants and the $C$. glabrata estimated genome size. These transformants were replica-printed onto SC-ura agar plates and 8 transformants were identified for their ability to grow on this medium. To rescue the plasmids from the transformants, total genomic DNA was prepared by the rapid glass bead procedure (Ausubel et al., 1987) and used to transform $E$. coli XL-1 blue competent cells to ampicillin resistance. Restriction analysis of plasmid DNA from one uracil prototroph indicated that this plasmid, designated U1(b), contained a $3.7-\mathrm{kb}$ fragment and that this plasmid can complement the ura $3^{-}$phenotype when re-transformed into DTY7 (MATa hist lou2-3, -112 ura3-52). To locate the putative $C$. glabrata URA3 gene within this fragment, fragments of the original $3.7-\mathrm{kb}$ insert were subcloned into pRS425 and transformed into DTY7 for complementation analysis. A $1-\mathrm{kb}$ $X h o I+S s p l$ fragment was identified that enabled DTY 7 cells to grow on SC-ura media (a). To determine the nucleotide sequence of the $C$. glabrata URA3 gene, fragments of the 1021-bp Xhol + Sspl insert were subcloned into the plasmid pBluescript $\mathrm{SK}(+)$ as indicated. Plasmid DNA was prepared and dideoxy sequencing of the double-stranded plasmid DNA was carried out using protocols described in the Sequenase Version 2.0 guidebook from US Biochemical (Cleveland, OH, USA). The primers used for sequencing both strands of DNA inserts are the M13-20 primer (5'-GTAAAACGACGGCCAGT) and T3 primer (5-ATTAACCCTCACTAAAG), which hybridize to either ends before the inserted DNA fragments (see Stratagene catalog). Positions of the nt encompassing the $C$. glabrata URA3 gene are numbered relative to the first $n t(+1)$ in the URA3 ORF (b). The deduced aa are shown under the first nt of each codon. The GenBank accession No. is L13661. 
chromosomal, self-replicating plasmid. Furthermore, transformants with pRS316 can be cured of the plasmid by selection on 5-FOA medium. All isolates resistant to 5-FOA revert to uracil auxotrophy, as expected, consistent with a lack of integration of the plasmid into the $C$. glabrata genome (data not shown).

The copy number of the pRS316 plasmid was determined in two C. glabrata strains: $\mathrm{Q}$ and the amt $1-1$ strain, in which the $C$ glabrata $A M T 1$ copper-activated transcription factor gene was insertionally inactivated (Zhou et al., 1992). By comparing the counts (cpm) of the linearized plasmid bands to the authentic $A M T 1$ gene DNA restriction fragments in Southern blotting analysis, we calculated the ratios from each comparison, which indicate that pRS316 is maintained al from 10 to 31 copies in Q or amt1-1 cells (Fig. 3). These results confirm that the pRS316 plasmid retains episomal replication function in C. glabrata, although it is currently unclear why the single copy $S$. cerevisiae plasmid, pRS316, exists as a highcopy plasmid in C. glabrata. Another episomal plasmid, $\mathrm{pW} 25$, that contains multiple copies of the MT-IIa gene on the plasmid pRS426 (Sikorski and Hieter, 1989), was constructed and also found to be able to replicate in the C. glabrata $\mathrm{Q}$ strain. The existence of a weak autonomously replicating sequence $A R S$ activity associated with the MT-IIa gene was recently reported (Mehra et al., 1992). However, the small colony size of transformants harboring only one copy of the MT-IIa gene on pRS426 suggests that the MT-IIa gene has poor ARS function relative to that of pRS316. The $S$. cerevisiae high copy plasmid pRS426, which contains $2 \mu$ plasmid DNA sequences, was also transformed into the C. glabrata $\mathrm{Q}$ strain. The recipient cells grew as microcolonies on SC-ura plates, indicating that pRS426 replicates poorly in $C$. glabrata. This suggests that the $S$. cerevisiae $2 \mu$ sequence can not foster efficient replication in C. glabrata. The existence of extra-chromosomal $2 \mu$-like circular DNA has not been reported in C. glabrata.

\section{(c) Integrative transformation and homologous recombination at the $C$. glabrata ura 3 locus}

Since the plasmid U1(b) can not self-replicate in $C$. glabrata, we used it as an integrative plasmid to target integration of genes at the C. glabrata ura 3 locus (Fig. 4a). Following integrative transformation using the $A M T 1$ gene carried on the linearized U1(b) plasmid, our Southern blotting analysis detected the appearance of the diagnostic $10.7-\mathrm{kb}$ (Pst I digestion) or $2.2-\mathrm{kb}$ and $0.7-\mathrm{kb}$ (StuI +SpeI digestion) restriction fragments of the $U R A^{+}$ transformant (Fig. 4b, c), indicating that U1(b) plasmid has successfully delivered the $A M T 1$ gene to the $C$. glabrata ura 3 locus. Two pRS425-based integrative plasmids with the $U R A 3$ gene carried on different DNA restriction

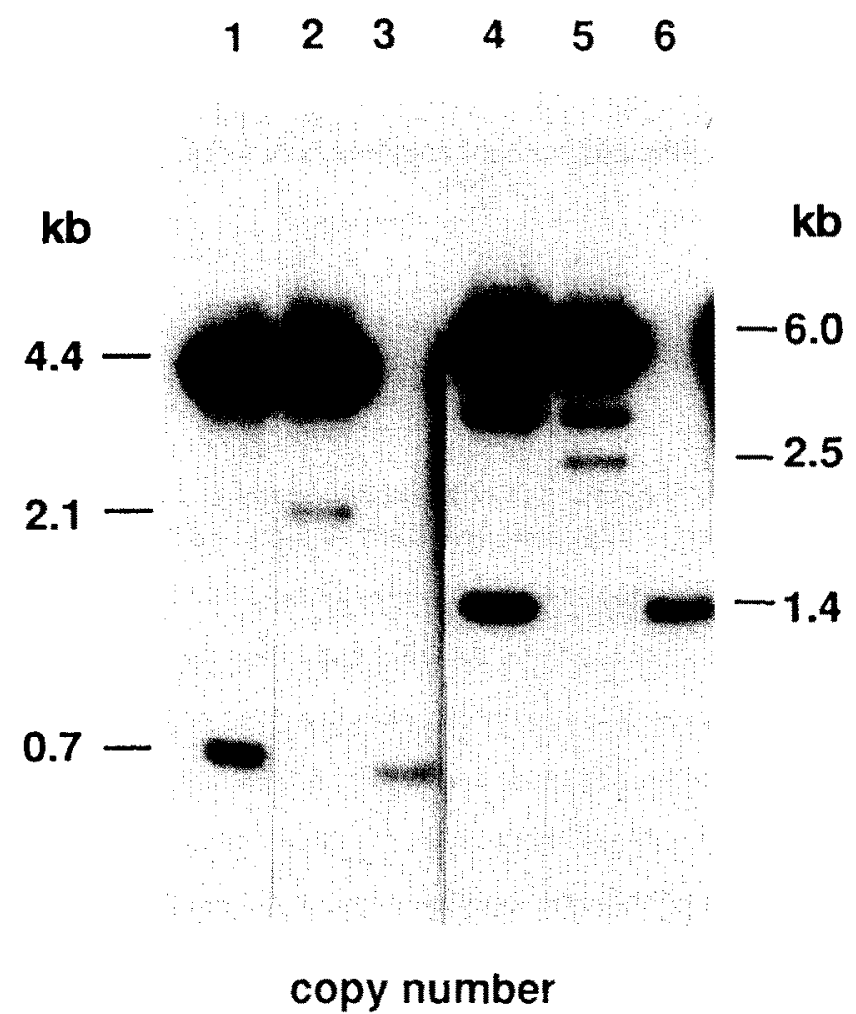

$\begin{array}{llll}20 & 31 & 10 & 22\end{array}$

Fig. 3. Southern blotting analysis to determine the copy number of the pRS316 plasmid in C. glabrata. The C. glabrata Q (lane 1 and 4) and amt 1-1 (Zhou et al., 1992) (lanes 2 and 5) strains were transformed with the pRS316 plasmid that contained a 613-bp BglII-StyI fragment of the $A M T 1$ gene ( $5^{\prime}$ portion) which was fused in-frame to the $E$. coli lac $Z$ gene. Genomic DNA from the recipient cells, or from the $Q$ cells without episomal plasmid (lanes 3 and 6 ) was prepared and digested to completion with the restriction enzymes $X$ hal $+X h o l+B g l l l(l a n e s ~ 1-3)$ or $P s t I+B g i I$ (lanes $4-6)$. Southern blotting $\left(1.0^{\%} \%\right.$ agarose gel) was carried out as described (Ausubel et al., 1987) using a 613-bp ${ }^{32}$ P-labeled $B g / I-S t y l$ fragment containing the $5^{\prime}$ portion of the $A M T I$ gene as the hybridization probe (Zhou and Thiele, 1991). The copy number of the plasmid was determined by comparing respectively, the cpm of ${ }^{32} \mathrm{P}$ associated with the 4.4-kb or 6.0 -kb plasmid DNA fragments with that of the chromosomal $A M T 1$ bands $(0.7$ or $1.4 \mathrm{~kb}$ in the Q strain. 2.1 or $2.5 \mathrm{~kb}$ in the amt $1-1$ strain) using a Beta-gen scanner. The sizes of each fragment (in $\mathrm{kb}$ ) corresponding to the extrachromosomal plasmid and the authentic $A M T I$ gene restriction fragments are indicated. The number below each lane represents the copy number of the pRS316 plasmid, relative to the single endogenous $A M T 1$ locus.

fragments were tested for their efficiency of integrative transformation and homologous recombination. The plasmid carrying the $3.7-\mathrm{kb}$ Sau3A fragment [U1(b)] transformed more efficiently than that containing only a $1.0-\mathrm{kb} X$ XoI-SspI fragment which only encompasses the $U R A 3$ ORF. This observation suggests a positive correlation between the length of homologous DNA and efficient chromosomal recombination in C. glabrata, however, further experimentation must be conducted to ascertain the validity of this observation. 


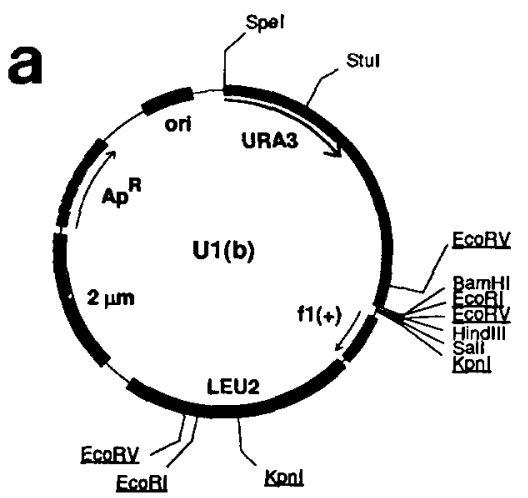

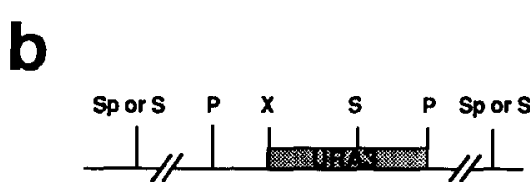
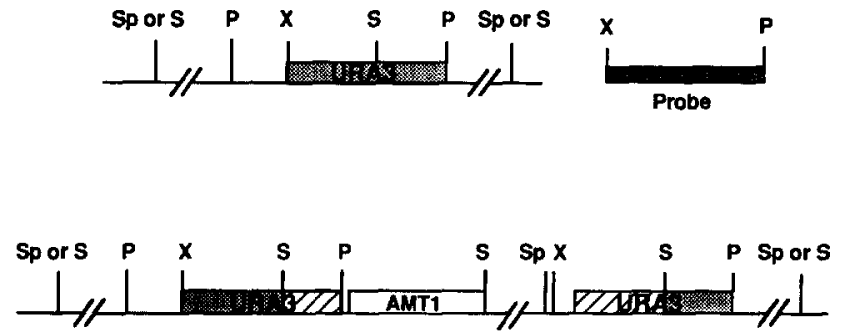

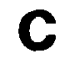

$\begin{array}{llll}1 & 2 & 3 & 4\end{array}$

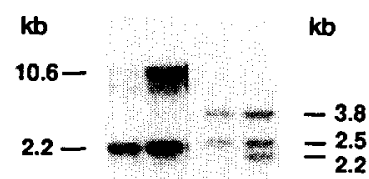

$-0.7$

Fig. 4. Gene targeting at the $C$. glabrata ura3 locus. (a) C. glabrata integrative plasmid U1(b). A 3.7-kb C. glabrata genomic fragment containing the URA3 gene was cloned into the BamHI site of the plasmid pRS425 to make the plasmid U1(b) (10.6-kb). The restriction sites that can be used for subcloning genes of interest into the U1(b) plasmid are indicated. The $E c o R I, E c o R V$ and $K p n I$ sites underlined are not unique but can also be used as cloning sites, resulting in the inactivation of the LEU2 gene. Prior to transformation, the plasmid may be linearized by digestion with Stul, which has a unique restriction site within the $U R A 3$ coding sequence, to facilitate homologous recombination. (b) Schematic representation of the $U R A 3$ locus prior and after plasmid integration. (c) Southern blotting verified the integration. Integrative plasmid UL(b), which carries the $A M T 1$ gene, was first digested to completion with $S t u l$. The linearized plasmid was transformed into Q. Genomic DNA from Q or a $U R A^{+}$transformant were digested to completion with PstI (lanes 1 and 2) or StuI + Spel (lanes 3 and 4), and fractionated on a $1 \%$ agarose gel. Southern blotting was performed (Ausubel et al., 1987) using a 1.5 -kb ${ }^{32} \mathrm{P}$-labeled $X$ hol + PstI DNA restriction fragment encompassing the URA3 ORF as a hybridization probe. The sizes of each DNA restriction fragment are indicated in kb. P, Pstl; S, StuI; Sp, Spel; X, XhoI.

\section{(d) Conclusions}

(1) An auxotrophic strain of $C$. glabrata, carrying a non-functional ura3 locus was isolated by EMS mutagenesis from the wt strain $85 / 038$.

(2) The C. glabrata URA3 gene was cloned and sequenced. It encodes a 265 -aa polypeptide which is highly similar to the $U R A 3$ gene product from the baker's yeast $S$. cerevisiae and other yeasts. The $U R A 3$ gene provides a defined genetic locus for targeted gene integration in C. glabrata.

(3) The episomal plasmid pRS316, initially constructed as a low copy replicating plasmids in $S$. cerevisiae, was demonstrated to replicate efficiently in the C. glabrata $u r a 3^{-}$strain. This plasmid can shuttle between C. glabrata, S. cerevisiae and E. coli, and should prove useful for gene manipulations or phylogenetic studies in these two yeast systems.

(4) Modified or wt genes of interest which are cloned into the URA3-based integrative plasmids, such as U1(b), can be efficiently targeted to integrate at the $C$. glabrata ura3 locus for genetic and functional analysis. Gene disruption, deletion and replacement can be achieved by constructing integrative plasmids or specific DNA restriction fragments carrying genes of interest and the appropriate selection marker such as $U R A 3$.

\section{ACKNOWLEDGEMENTS}

We thank Simon Knight for critical reading of the manuscript and Paul Joyce and Nancy Martin for helpful discussions and encouragement. This research was funded by a National Institutes of Health grant GM41840 and grant M01-RR00042 to the General Clinical Research Center, University of Michigan from the NIH. P.Z. was supported in part by a Rackham Predoctoral fellowship from the Horace $H$. Rackham School of Graduate Studies, University of Michigan, and a Loeb Predoctoral fellowship from the University of Michigan Cancer Center.

\section{REFERENCES}

Aisner, I., Schimpff, S.C.. Sutherland, J.C., Young, V.M. and Wiernik, P.H.: Torulopsis glabrata infections in patients with cancer. Increasing incidence and relationship to colonization. Am. J. Med. 61 (1976) 23-28.

Ausubel, F.M., Brent, R., Kingston, R.E., Moore, D.D., Seidman, J.A., Smith, J.A. and Struhl, K. (Eds.): Current Protocols in Molecular Biology. Wiley, New York, 1987.

Barns, S.M., Lane, D.J.. Sogin. M.L.. Bibeau. C. and Weisburg. W.G.: Evolutionary relationships among pathogenic Candida species and relatives. J. Bacteriol. 173 (1991) 2250-2255.

Damani, N.N. and Webb, C.H.: Tortulopsis glabrata fungaemia. Ulster Med. J. 57 (1988) 220-223.

Ernst, J.F. and Losberger, C.: Sequence and transeript analysis of the C. albicans URA3 gene encoding orotidine- $5^{\prime}$-monophosphate decarboxylase. Curr. Genet. 16 (1989) 153-157.

Ferrante A.: Tumor necrosis factor alpha potentiates neutrophil antimicrobial activity: increased fungicidal activity against Torulopsis glabrata and candida albicans and associated increases in oxygen radical production and lysosomal enzyme release. Infect. Immun. 57 (1989) 2115-2122.

Frye, K.R., Donovan, J.M. and Drach, G.W.: Torulopsis glabrata urinary infections: a review. J. Urol. 139 (1988) 1245-1249.

Haas, L.O.C., Cregg, J.M. and Gleeson, M.A.G.: Development of an integrative DNA transformation system for the yeast Candida tropicalis. J. Bacteriol. 172 (1990) 4571-4577. 
Hickey, W.F., Sommerville, L.H. and Schoen, F.J.: Disseminated Candida glabrata: report of a uniquely severe infection and a literature review. Am. J. Clin. Pathol. 80 (1983) 724-727.

Mehra. R.K., Thorvaldsen. J.L., Macreadie, I.G. and Winge, D.R.: Cloning system for Candida glabrata using elements from the metallothionein- $\mathrm{II}_{\mathrm{a}}$-encoding gene that confer autonomous replication. Gene 113 (1992) 119-124.

Moniaci, D., Roggia, S., Giacomettiu. E., Sinicco, A. and Re, G.: A rare case of Torulopsis glabrata in a patient with AIDS. Minerva Stomatol. 37 (1988) 343-346.

Odds, F.C.: Candida infections: an overview. CRC Crit. Rev. Microbiol 15 (1987) $1-5$

Redondo-Lopez, V., Lynch, M., Schmitt, C., Cook, R. and Sobel, J.D.: Torulopsis glabrata vaginitis: clinical aspects and susceptibility to antifungal agents. Obstet. Gynecol. 76 (1990) 651-655.

Rose, M., Grisafi, P. and Bostein, D.: Structure and function of the yeast URA3 gene: expression in Escherichia coli. Gene 29 (1984) 113-124.

Scherer, S. and Magee, P.T.: Genetics of Candida albicans. Microbiol. Rev. 54 (1990) 226-241.

Sherman, F., Fink, G.R. and Hicks, J.B.: Methods in Yeast Genetics Cold Spring Harbor Laboratory. Cold Spring Harbor, NY, 1983.
Shuster, J.R., Moyer, D. and Irvine, B. Sequence of the Kluyeromyces lactis URA3 gene. Nuclcic Acids Res. 15 (1987) 8573.

Sikorski, R.S. and Hieter, P.: A system of shuttle vectors and yeast host strains designed for efficient manipulation of DNA in Saccharomyces cerevisiae. Genetics 122 (1989) 19-27.

Sinnott, IV, J.T., Cullison, J.P. and Sweeney, M.P.: Candida (Torulopsis) glabrata. Infect. Control 8 (1987) 334-336.

Whelan, W.L.: The genetics of medically important fungi. CRC Crit Rev. Microbiol. 14 (1987) 99-140.

White, R.W.: Ethanol fermentation of glucose by Torulopsis glabrata in the stomachs of neonates of the horse, dog, goat and Soay sheep. Br. Vet. J. 132 (1976) 654-656.

Zhou, P. and Thiele. D.J.: Isolation of a metal-activated transcription factor gene from Candida glabrata by complementation in Saccharomyces cerevisiae. Proc. Natl. Acad. Sci. USA 88 (1991) 61126116.

Zhou, P., Szczypka, M.S., Sosinowski, T. and Thiele, D.J.: Expression of a yeast metallothionein gene family is activated by a single metalloregulatory transcription factor. Mol. Cell. Biol. 12 (1992) $3766-3775$. 